\title{
Optimal Control of a Ball and Beam Nonlinear Model Based on Takagi-Sugeno Fuzzy Model
}

\author{
José Miguel Adánez \\ Fernande Matía \\ Basil Møhammed Al-Hadithi Agustín Jiménez
}

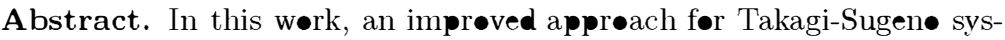
tem identification is used. Linear Quadratic Regulat•r is applied før an -ptimal state feedback. Duality theørem and Linear Quadratic Regula-

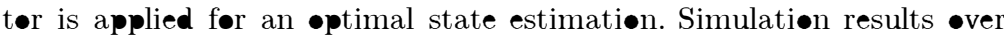

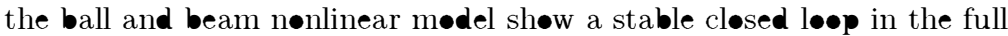
range and gøod transient response.
\end{abstract}

\section{Introduction}

The ball and beam system [1] is a classical mechanical system with tw॰ degrees - freedom. The beam rotates, driven by a torque at the center of rotation. The ball rells freely aløng the beam and in contact with the beam. Despite its mechanical simplicity, the ball and beam system presents significant challenges frøm the point of view of aut॰mation; the system is nønlinear and unstable.

The ball and beam is a common didactical plant in many contrøl laborateries arøund the world [2], as it is very nonlinear, unstable, which means that it is

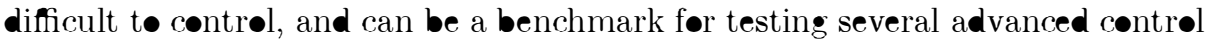
techniques [3].

Takagi-Sugen• (T-S) fuzzy mødel [4] has been an important tool for the mødelling and contrøl of nonlinear systems, since it builds the full nønlinear mødel by a linear model at each fuzzy rule and the fuzzy interpolation amøn: them. Møreøver, the T-S fuzzy identification alløws the identification of all the fuzzy parameters of the full nønlinear system minimizing a gløbal errør index.

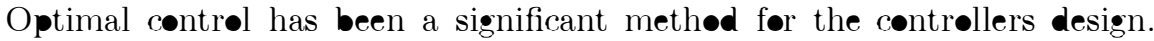

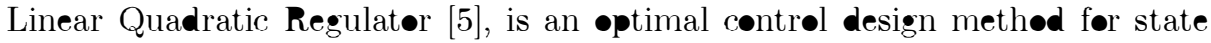
space linear models which alløws the minimization of a cost function in which state dynamics and contrøl actiøn are weighted. 
One of the most important problems in state space feedback is that usually the states are not directly accessible, since not all the state variables are measurable. For this propose, state observers [6], can create a surrogate state vector, which tends asymptotically to the real state vector and can be used for state feedback.

The duality theorem [6] allows the design of an state observer matrix with the same techniques for an state feedback controller, including the LQR method [5]. For that propose a dual system can be built from the state space model, and the duality theorem says that a controller designed in the dual system is equivalent to an observer designed for the state space model.

The rest of the work is organized as follows. Section 2 describes ball and beam nonlinear model. The fuzzy T-S model and the fuzzy identification method are described in Sect.3. Optimal state controller and optimal state observer designs are described in Sect. 4 . In Sect. 5, the proposed fuzzy optimal controller is applied to the ball and beam nonlinear model and the results are obtained and discussed.

\section{Ball and Beam Nonlinear Model}

In this work, we use the AMIRA BW500 ball and beam model (Fig. 1) [1]. The ball position $p$, considered as system output, is supposed to be measured by a camera, therefore the discrete sample time is supposed to be large. The beam angle $\alpha$, considered as measurable internal variable, is supposed to be measured by an incremental encoder. The system input $F$ is supposed to be a force produced by a DC motor, which causes the beam to rotate around its center.

The nonlinear differential equations of the ball and beam model [1], used for the simulation model, are:

$$
\begin{gathered}
\left(m+\frac{I_{b}}{r^{2}}\right) \ddot{p}+\left(m r^{2}+I_{b}\right) \frac{1}{r} \ddot{\alpha}-m p \dot{\alpha}^{2}=m g \sin (\alpha) \\
\left(m p^{2}+I_{b}+I_{W}\right) \ddot{\alpha}+\left(2 m p \dot{p}+b l^{2}\right) \dot{\alpha}+K l^{2} \alpha+ \\
\left(m r^{2}+I_{b}\right) \frac{1}{r} \ddot{p}-m g p \cos (\alpha)=F l \cos (\alpha)
\end{gathered}
$$

where $p$ is the position of the ball, $\alpha$ is the angle of the beam and $F$ is the force of the drive mechanics. Table 1 summarizes the parameters of the model and its values.

In this model, some restrictions from the real AMIRA BW500 ball and beam model [1] have to be added. The ball position $p$ has to be contained in $[-0.4,0.4] m$, the beam angle $\alpha$ has to be contained in $[-0.69,0.69] \mathrm{rad}$ and the input force $F$ has to be contained in $[-5,5] \mathrm{N}$.

Since we consider $\alpha$ just as a measurable internal variable, the system is single-input-single-output (SISO), and the relation between $p$ as output and $F$ as input is described by two second order differential equations, so the global system is fourth order. 


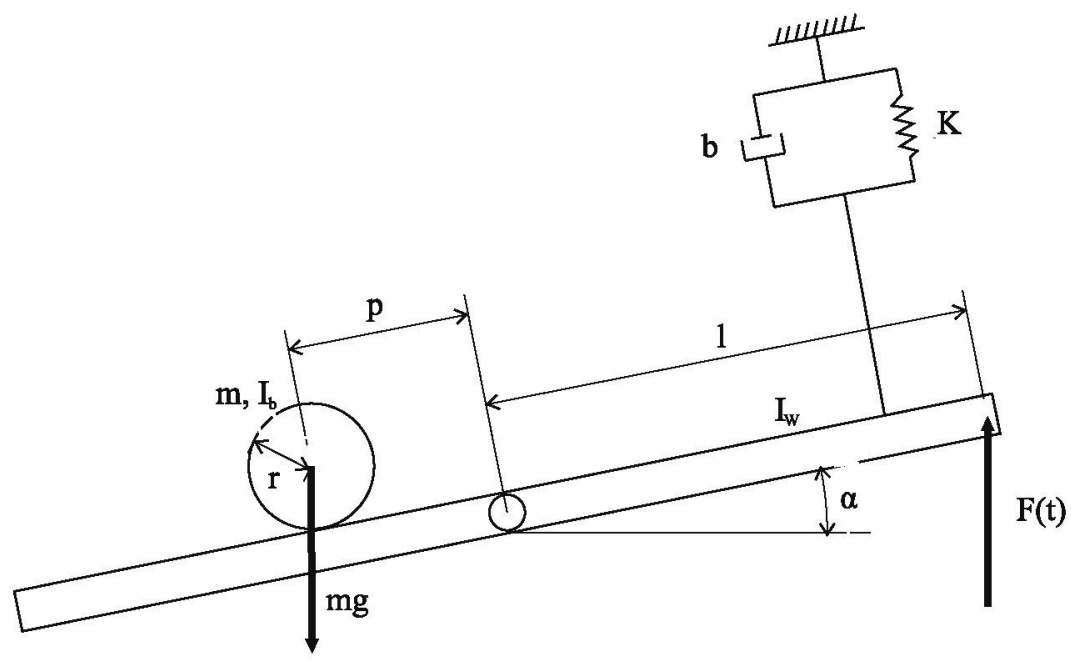

Fig. 1. Ball and beam system.

Table 1. Ball and beam parameters

\begin{tabular}{l|l|l}
\hline Parameter & Meaning & Value \\
\hline$m$ & Mass of the ball & $0.025 \mathrm{Kg}$ \\
\hline$g$ & Gravity & $9.81 \mathrm{~m} / \mathrm{s}^{2}$ \\
\hline$r$ & Roll radius of the ball & $0.0167 \mathrm{~m}$ \\
\hline$I_{b}$ & Inertia moment of the ball & $3.516 \cdot 10^{-6} \mathrm{Kgm}^{2}$ \\
\hline$I_{W}$ & Inertia moment of the beam & $0.09 \mathrm{Kgm}$ \\
\hline$b$ & Friction coefficient of the drive mechanics & $1.0 \mathrm{Ns} / \mathrm{m}$ \\
\hline$K$ & Stiffness of the drive mechanics & $0.001 \mathrm{~N} / \mathrm{m}$ \\
\hline$l$ & Radius of force application & $0.49 \mathrm{~m}$ \\
\hline
\end{tabular}

\section{Fuzzy Takagi-Sugeno Model and System Identification}

\subsection{Fuzzy T-S Model}

Nonlinear systems can be modelled by T-S model, supposing known a set of measurable nonlinear variables $\left[z_{1}(k), z_{2}(k), \ldots, z_{m}(k)\right]$ of the system. By choosing $\left[r_{1}, r_{2}, \ldots, r_{m}\right]$ number of fuzzy sets for these variables, a monovariable fuzzy system can be defined as follows:

$$
\begin{aligned}
& S^{\left(i_{1} \ldots i_{m}\right)}: \text { If } z_{1}(k) \text { is } M_{1}^{i_{1}} \text { and } \ldots \text { and } z_{m}(k) \text { is } M_{m}^{i_{m}} \text { then: } \\
& \qquad \begin{array}{l}
y(k)=a_{0}^{\left(i_{1} \ldots i_{m}\right)}+a_{1}^{\left(i_{1} \ldots i_{m}\right)} y(k-1)+\cdots+a_{n}^{\left(i_{1} \ldots i_{m}\right)} y(k-n) \\
+b_{1}^{\left(i_{1} \ldots i_{m}\right)} u(k-1)+\cdots+b_{n}^{\left(i_{1} \ldots i_{m}\right)} u(k-n)
\end{array}
\end{aligned}
$$


In each rule, we can transform the difference Eq. (3) to state model with affine term as follows:

$$
\begin{aligned}
& S^{\left(i_{1} \ldots i_{m}\right)} \text { : If } z_{1}(k) \text { is } M_{1}^{i_{1}} \text { and } \ldots \text { and } z_{m}(k) \text { is } M_{m}^{i_{m}} \text { then: } \\
& x(k) \in \mathfrak{R}^{n} \\
& x(k+1)=\left[\begin{array}{cc}
a_{0}^{\left(i_{1} \ldots i_{m}\right)} \cdot a_{1}^{\left(i_{1} \ldots i_{m}\right)} \\
a_{0}^{\left(i_{1} \ldots i_{m}\right)} \cdot a_{2}^{\left(i_{1} \ldots i_{m}\right)} \\
\vdots \\
a_{0}^{\left(i_{1} \ldots i_{m}\right)} \cdot a_{n}^{\left(i_{1} \ldots i_{m}\right)}
\end{array}\right]+ \\
& +\left[\begin{array}{cccc}
a_{1}^{\left(i_{1} \ldots i_{m}\right)} & 1 & \ldots & 0 \\
a_{2}^{\left(i_{1} \ldots i_{m}\right)} & 0 & \ddots & 0 \\
\vdots & \vdots & \ddots & 1 \\
a_{n}^{\left(i_{1} \ldots i_{m}\right)} & 0 & \cdots & 0
\end{array}\right] x(k)+\left[\begin{array}{c}
b_{1}^{\left(i_{1} \ldots i_{m}\right)} \\
b_{2}^{\left(i_{1} \ldots i_{m}\right)} \\
\vdots \\
b_{n}^{\left(i_{1} \ldots i_{m}\right)}
\end{array}\right] u(k) \\
& y(k)=a_{0}^{\left(i_{1} \ldots i_{m}\right)}+\left[\begin{array}{llll}
1 & 0 & \cdots & 0
\end{array}\right] x(k)
\end{aligned}
$$

This means

$$
\begin{aligned}
& S^{\left(i_{1} \ldots i_{m}\right)} \text { : If } z_{1}(k) \text { is } M_{1}^{i_{1}} \text { and } \ldots \text { and } z_{m}(k) \text { is } M_{m}^{i_{m}} \text { then: } \\
& x(k+1)=a_{x}^{\left(i_{1} \ldots i_{m}\right)}+A^{\left(i_{1} \ldots i_{m}\right)} x(k)+B^{\left(i_{1} \ldots i_{m}\right)} u(k) \\
& y(k)=a_{y}^{\left(i_{1} \ldots i_{m}\right)}+C x(k)
\end{aligned}
$$

\subsection{Estimation of T-S Model Parameters}

The identification method of T-S fuzzy models [4] is based on the estimation of the fuzzy system parameters minimizing a quadratic performance index. The traditional T-S identification method [4] fails if the membership functions of the fuzzy rules are overlapped triangular in shape, since the T-S matrix is not of full rank and then it is not invertible [7]. Thus, in [7] was proposed a generalized T-S identification, using a parameters weighting method.

The fuzzy estimation of the output becomes:

$$
\begin{aligned}
& \hat{y}=\sum_{i_{1}=1}^{r_{1}} \cdots \sum_{i_{m}=1}^{r_{m}} \beta^{\left(i_{1} \ldots i_{m}\right)}\left(z_{\left(i_{1} \ldots i_{m}\right)}(k)\right)\left[a_{0}^{\left(i_{1} \ldots i_{m}\right)}+a_{1}^{\left(i_{1} \ldots i_{m}\right)} y(k-1)\right. \\
& \left.+\cdots+a_{n}^{\left(i_{1} \ldots i_{m}\right)} y(k-n)+b_{1}^{\left(i_{1} \ldots i_{m}\right)} u(k-1)+\cdots+b_{n}^{\left(i_{1} \ldots i_{m}\right)} u(k-n)\right]
\end{aligned}
$$

where

$$
\beta^{\left(i_{1} \ldots i_{m}\right)}\left(z_{\left(i_{1} \ldots i_{m}\right)}(k)\right)=\frac{\mu_{1 i_{1}}\left(z_{1}\right) \ldots \mu_{m i_{m}}\left(z_{m}\right)}{\sum_{i_{1}=1}^{r_{1}} \cdots \sum_{i_{m}=1}^{r_{m}}\left(\mu_{1 i_{1}}\left(z_{1}\right) \ldots \mu_{m i_{m}}\left(z_{m}\right)\right)}
$$

with $\mu_{j i_{j}}\left(z_{j}\right)$ being the membership function corresponding to the fuzzy set $M_{j}^{i_{j}}$. 
We have supposed to have a set of input/output system samples and a first affine linear parameters estimation:

$$
p^{0}=\left[a_{0}^{0} a_{1}^{0} \ldots a_{n}^{0} b_{1}^{0} \ldots b_{n}^{0}\right]
$$

These parameters could be obtained by a classical input/output identification of the data, for example with least squares method. This first approximation can be utilized as reference parameters for all the subsystems. Then, the fuzzy model parameters can be obtained minimizing:

$$
\begin{aligned}
& J=\sum_{k=1}^{s}(y(k)-\hat{y}(k))^{2}+\gamma^{2} \sum_{i_{1}=1}^{r_{1}} \cdots \sum_{i_{m}=1}^{r_{m}} \sum_{j=0}^{n}\left(p_{j}^{0}-p_{j}^{\left(i_{1} \ldots i_{m}\right)}\right)^{2} \\
& =\|Y-X P\|^{2}+\gamma^{2}\left\|P_{0}-P\right\|^{2}=\left\|\left[\begin{array}{c}
Y \\
\gamma P_{0}
\end{array}\right]-\left[\begin{array}{c}
X \\
\gamma I
\end{array}\right] P\right\|^{2}=\left\|Y_{a}-X_{a} P\right\|^{2}
\end{aligned}
$$

where $Y$ are the output data, $X$ are the input/output fuzzy data, $P_{0}$ are the linear estimated parameters repeated as many times as the number of fuzzy rules $\left(P_{0}=\left[p_{0}, p_{0}, \ldots, p_{0}\right]\right)$, and $P$ are the fuzzy T-S model parameters. The $\gamma$ factor represents the degree of confidence of the linear estimated parameters, and it must be tuned by try and error. It should be noted that the matrix $X_{a}$ is of full rank, which solves the problem where the traditional T-S identification method fails. Thus, the vector $P$ can be computed as:

$$
P=\left(X_{a}^{t} X_{a}\right)^{-1} X_{a}^{t} Y_{a}
$$

\section{Fuzzy Controller and Observer Design}

In order to calculate the coefficients of the state feedback controller, discrete LQR [5] method is chosen, which allows optimal control weighting the dynamic response and the control action.

In LQR method, the goal is to minimize the cost index $\mathrm{J}$ :

$$
J=\sum_{k=0}^{\infty}\left[\left(x(k)-x_{r}\right)^{t} Q\left(x(k)-x_{r}\right)+\left(u(k)-u_{r}\right)^{t} R\left(u(k)-u_{r}\right)\right]
$$

LQR method is completely optimal for linear systems, however, in the case of nonlinear systems, it is complex to propose the minimization of any objective function for the global system. In order to solve this problem, we suggest minimizing the cost of each fuzzy rule instead of the global cost. The solution will be a suboptimal one but with the great advantage of being easy to calculate. With this method, the global stability is not guaranteed, which needs to be analyzed a posteriori, although gaining in return a balance between static and dynamic behavior of the system with admissible control actions.

The state observer [6] is a parallel dynamic system with a correction term that approximates the estimated state to the real one:

$$
\begin{aligned}
x_{e}(k+1) & =a_{x}+A x_{e}(k)+B u(k)+H\left(y(k)-y_{e}(k)\right) \\
y_{e}(k) & =a_{y}+C x_{e}(k)
\end{aligned}
$$


The estimation error is:

$$
\begin{aligned}
\varepsilon(k+1) & =x(k+1)-x_{e}(k+1)=\left(a_{x}+A x(k)+B u(k)\right) \\
& -\left(a_{x}+A x_{e}(k)+B u(k)+H\left(y(k)-\left(a_{y}+C x_{e}(k)\right)\right)\right)
\end{aligned}
$$

which can be rewritten as follows:

$$
\varepsilon(k+1)=(A-H C) \varepsilon(k)
$$

The duality theorem [6] states that the design of a state observer is equivalent to designing a state feedback controller using some transformations in the state matrices. Based on the linear discrete system described as:

$$
\begin{aligned}
x(k+1) & =A x(k)+B u(k) \\
y(k) & =C x(k)
\end{aligned}
$$

The corresponding dual system becomes:

$$
\begin{aligned}
x_{d}(k+1) & =A_{d} x_{d}(k)+B_{d} u(k) \\
y_{d}(k) & =C_{d} x_{d}(k)
\end{aligned}
$$

where:

$$
\begin{aligned}
& A_{d}=A^{t} \\
& B_{d}=C^{t} \\
& C_{d}=B^{t}
\end{aligned}
$$

Therefore, it is possible to calculate a control matrix for the dual system $K_{d}$ equivalent to the observation matrix for the original system $H$ :

$$
H=K_{d}^{t}
$$

In this way, is possible calculate the observation matrix $H$, obtaining the controller matrix for the dual system $K_{d}$ by any state controller design method in the dual system. In this case, a discrete LQR [5] is proposed, obtaining the $H$ observer matrix from the dual system matrices $A_{d}=A^{t}$ and $B_{d}=C^{t}$, and the weighing matrices $Q_{d}$ and $R_{d}$, minimizing the following index cost:

$$
J=\sum_{k=0}^{\infty}\left[x_{d}(k)^{t} Q_{d} x_{d}(k)+u(k)^{t} R_{d} u(k)\right]
$$

\section{$5 \quad$ Results}

In this section, we apply the proposed fuzzy optimal controller to the ball and beam nonlinear model described in Sect. 2. The ball and beam model works in continuous time, but the controller has been developed in discrete time, so a sampler and zero order holding device have been added to the model, supposing the proposed sampling time is $T=0.05 \mathrm{~s}$. All system variables are supposed to be ideally measured. 
As first step, a linear identification of the system has been made by least squares method, obtaining a first affine linear parameters estimation:

$$
\begin{aligned}
p(k) & =0+3.92 p(k-1)-5.75 p(k-2)+3.75 p(k-3)-0.92 p(k-4) \\
& -0.0001 F(k-1)+0.0001 F(k-2)+0.0001 F(k-3)-0.0001 F(k-4)
\end{aligned}
$$

For a T-S model identification of the system, an iterative adjustment of the membership functions of the fuzzy rules and the weighting factor $\gamma$ have been made, adjusting by try and error the fuzzy membership functions defined in Fig. ? and the weighting factor $\gamma=3.7 \cdot 10^{-6}$, obtaining an identification error of $1.616910^{-11}$.

With the generalized T-S identification method and Eq. (4), a fuzzy T-S state matrices of the system has been obtained:

$$
\begin{aligned}
& S^{(1,1,1,1)} \text { : If } p(k) \text { is } M_{1}^{1} \text { and } \dot{p}(k) \text { is } M_{2}^{1} \text { and } \alpha(k) \text { is } M_{3}^{1} \text { and } \dot{\alpha}(k) \text { is } M_{4}^{1} \text { then: } \\
& a_{x}^{(1,1,1,1)}=10^{-5}[0.3146-0.46200 .3014-0.0737]^{t} \\
& A^{(1,1,1,1)}=\left[\begin{array}{cccc}
3.9181 & 1 & 0 & 0 \\
-5.7542 & 0 & 1 & 0 \\
3.7543 & 0 & 0 & 1 \\
-0.9181 & 0 & 0 & 0
\end{array}\right] \\
& B^{(1,1,1,1)}=10^{-3}[-0.05780 .14180 .1160-0.0544]^{t} \\
& a_{x}^{(1,1,1,1)}=8.0285 \cdot 10^{-7} \\
& C^{(1,1,1,1)}=\left[\begin{array}{llll}
1 & 0 & 0 & 0
\end{array}\right]
\end{aligned}
$$

Thus, the controller matrix $K$ is designed in each rule by discrete LQR [5] algorithm, using the system matrices $A$ and $B$, and the positive definite weighting matrices $Q=I$ and $R=1$. Obtaining the fuzzy controller matrix:

$$
\begin{gathered}
S^{(1,1,1,1)} \text { : If } p(k) \text { is } M_{1}^{1} \text { and } \dot{p}(k) \text { is } M_{2}^{1} \text { and } \alpha(k) \text { is } M_{3}^{1} \text { and } \dot{\alpha}(k) \text { is } M_{4}^{1} \text { then: } \\
K^{(1,1,1,1)}=10^{3}[2.95862 .54832 .17331 .8322]
\end{gathered}
$$

The observer algorithm is designed in each rule by duality theorem [6] and discrete LQR algorithm [5], using the dual system matrices $A_{d}=A^{t}$ and $B_{d}=$ $C^{t}$, and the positive definite weighting matrices $Q_{d}=I$ and $R_{d}=1$. Obtaining the fuzzy observer matrix by the Eq. (18):

$$
\begin{gathered}
S^{(1,1,1,1)} \text { : If } p(k) \text { is } M_{1}^{1} \text { and } \dot{p}(k) \text { is } M_{2}^{1} \text { and } \alpha(k) \text { is } M_{3}^{1} \text { and } \dot{\alpha}(k) \text { is } M_{4}^{1} \text { then: } \\
H^{(1,1,1,1)}=[2.7955-4.98733 .4842-0.8789]^{t}
\end{gathered}
$$

With this controller and observer design method, the global stability cannot be proved theoretically, so it has to be analyzed a posteriori with the simulation results. 

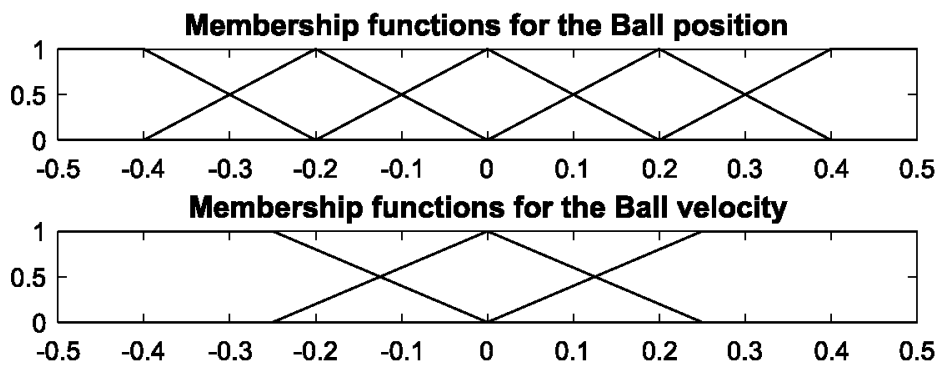

Membership functions for the Beam angle

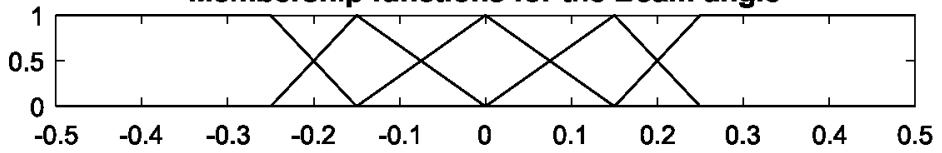

Membership functions for the Beam angular velocity

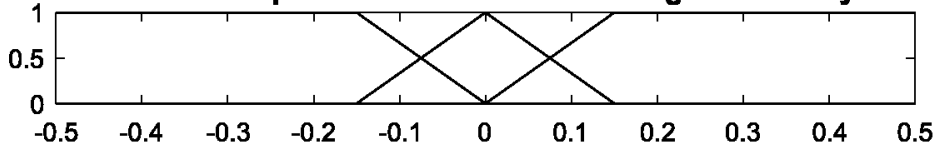

Fig. 2. Membership functions of the fuzzy sets.

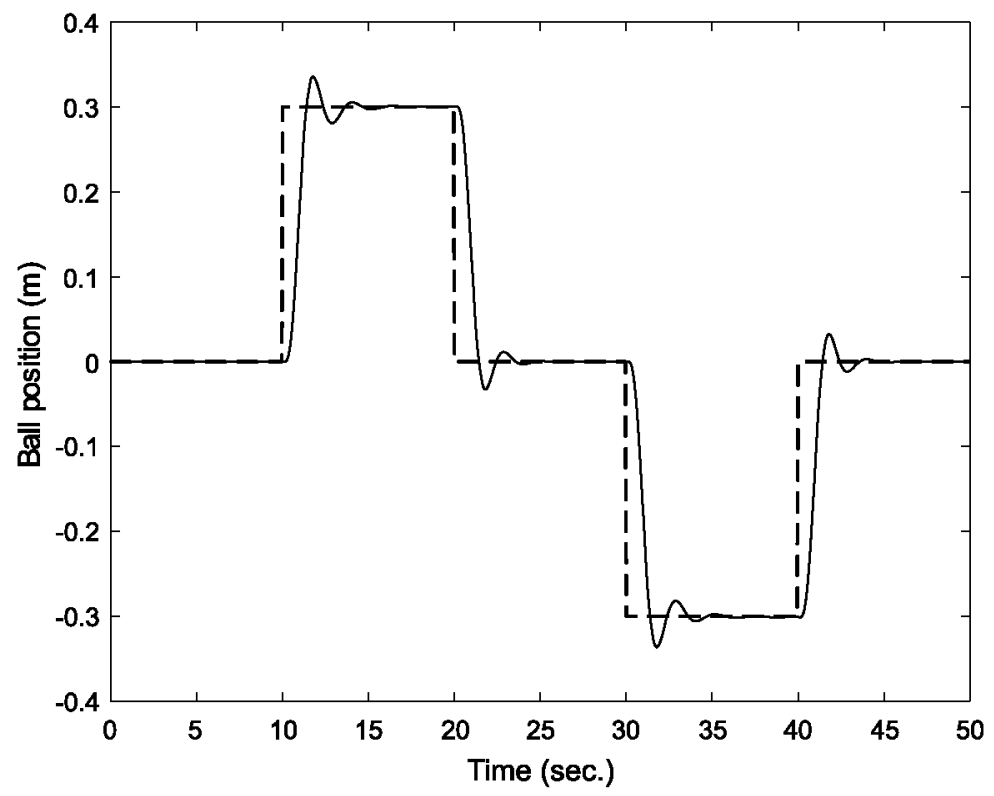

Fig. 3. Ball position.

Figures 3, 4, 5 and 6 show each one the position of the ball, the angle of the beam, the input force and the observation error of the ball position, when the controlled system is subjected to changes in the ball position reference. 


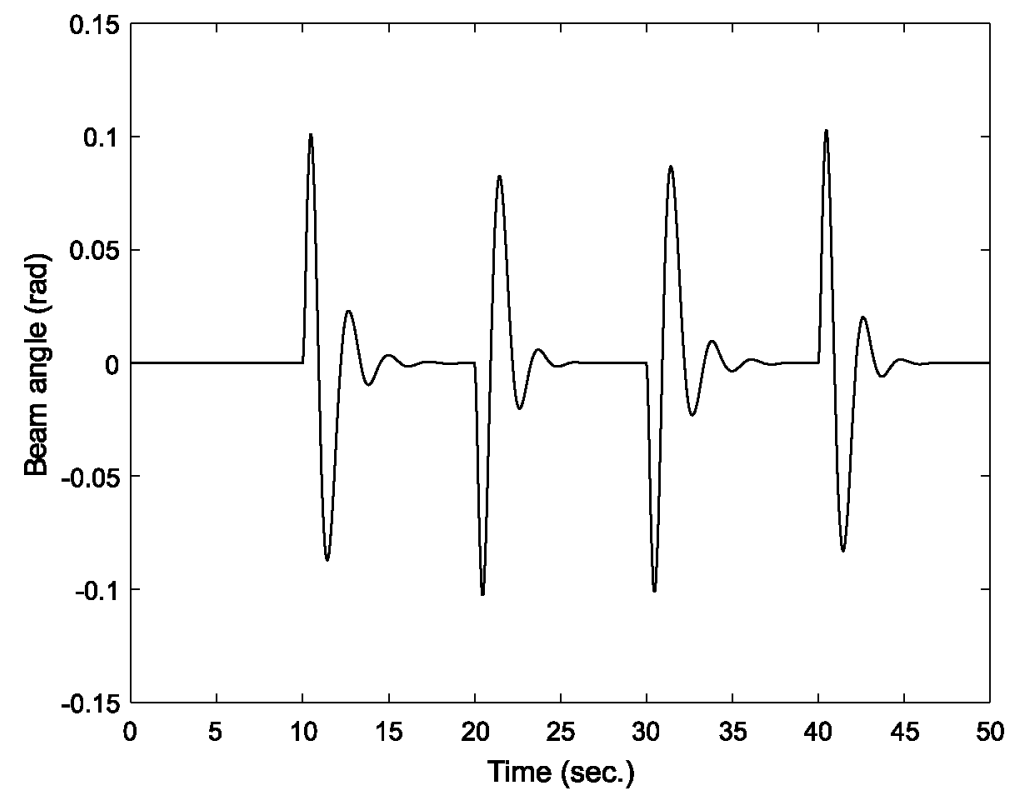

Fig. 4. Beam angle.

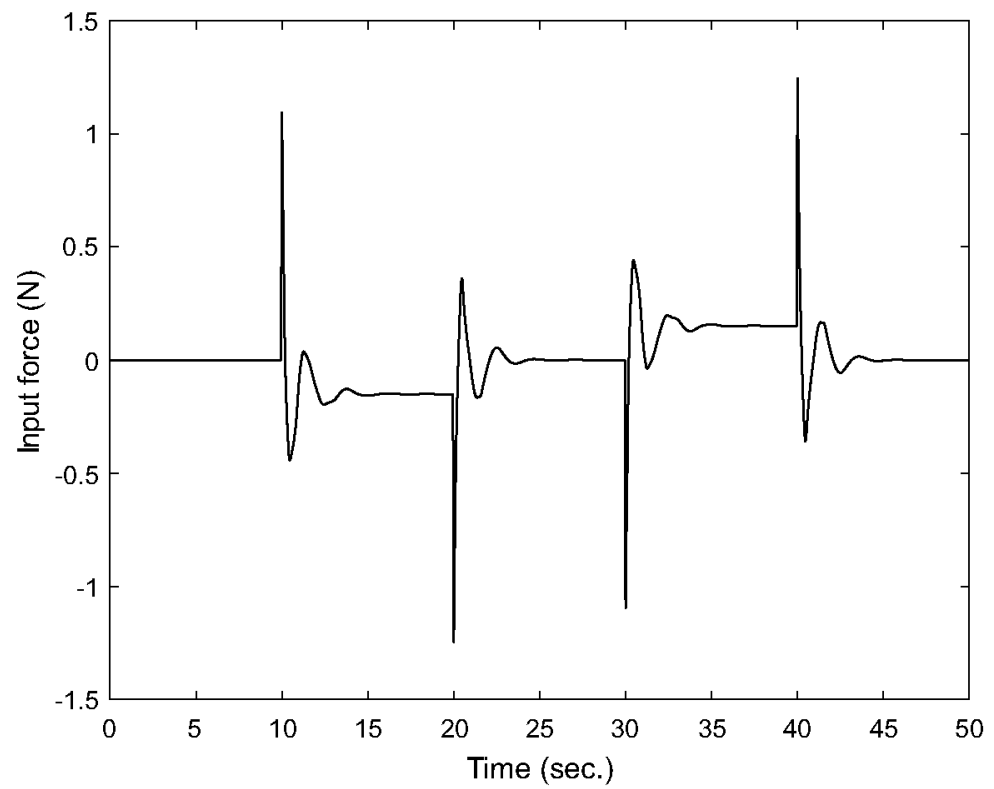

Fig. 5. Input force. 


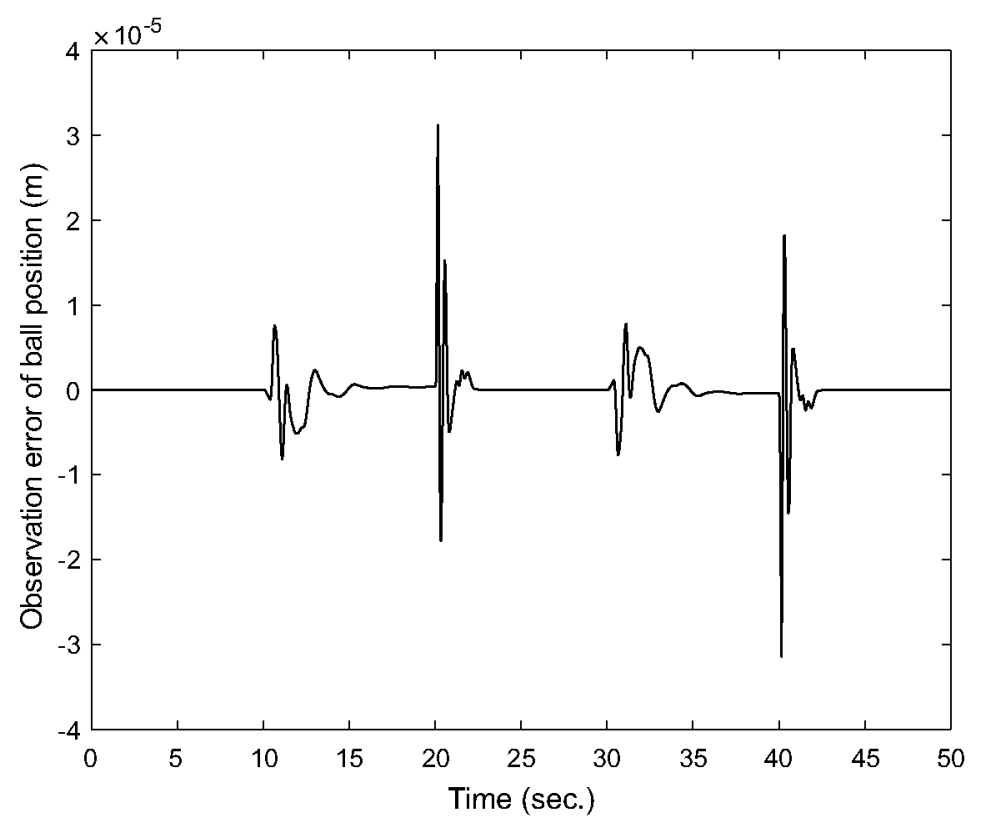

Fig. 6. Observation error of ball and beam position.

In Figs. 3, 4 and 5 it can be seen that, the system variables are in the physical range of the ball and beam, and all these variables present smooth and stable transient responses. In Fig. 6 it is shown that the observation error is small and tends to zero. Thus, the controlled ball and beam model has a stable response in the full range of the system and presents a good transient response.

\section{Conclusion}

In this work, we have shown the obtained results that a generalized T-S identification method and an optimal state controller and observer designed in each fuzzy rule, applied in a ball and beam nonlinear model. The fuzzy generalized T-S identification method is based on a weighting parameter of the previously estimated linear model. The optimal controller and observer has been designed in each fuzzy rule, so a suboptimal solution have been found, but easy of calculate and compute. The results show that the ball and beam controlled nonlinear model has a stable behavior and good transient response on the full range of the ball and beam system.

Aknowledgement. This work is funded by Spanish Ministry of Economy and Competitiveness (Assisted Navigation through Natural Language (NAVEGASE) project 265 (DPI 2014-53525-C3-1-R)). 